\title{
Uso de TIC y herramientas de aprendizaje activo para un curso de Física de Posgrado
}

\author{
Jonnathan Andre López Sánchez \\ jonnathan.lopez@unah.edu.hn \\ Escuela de Física \\ Universidad Nacional Autónoma de Honduras
}

¿.......

\section{Resumen}

En el presente artículo se comparte la experiencia y los resultados de la intervención educativa innovadora "Implementación en modalidad b-learning del módulo 2 de la asignatura Física Cuántica (MFS614)", que es parte de la Maestría de Física de la Facultad de Ciencias de la Universidad Nacional Autónoma de Honduras, UNAH.

Para esta intervención educativa, desarrollada en el primer período académico de 2016, se adoptó un enfoque de enseñanza conocido como spin-first, mediante el cual se introducen los principios de la mecánica cuántica moderna con experimentos de Stern-Gerlach.

Se utilizaron herramientas computacionales de vanguardia para la educación, tales como la versión abierta de la plataforma Open edX, una aplicación de la Universidad
Estatal de Oregon llamada SPINS para tratar la temática de manera interactiva, diferentes tutoriales escritos y en video que tienen como base dificultades de alumnos reportadas en universidades reconocidas a nivel mundial.

Entre los principales resultados obtenidos mediante la encuesta, el 80\% de los alumnos participantes resalta elevada satisfacción, quienes consideraron el enfoque utilizado más enriquecedor en cuanto a su aprendizaje, que otros meramente teóricos, como el ofrecido por el MIT a través de su plataforma MOOC edX. Además, una evaluación especial reveló que el formalismo y los conceptos fundamentales necesarios fueron bien asimilados por los alumnos. Estos resultados motivan a realizar más investigación en el área enseñanza-aprendizaje de este riguroso rubro de la física.
Palabras clave:

Mecánica Cuántica, Experimentos de Stern-Gerlach, entornos virtuales de aprendizaje, aprendizaje activo en STEM.

\section{Abstract}

This paper summarizes the experience and results of the innovative educational intervention "B-learning implementation for the second module of Quantum Physics course", which is part of the Physics Master at the Science Faculty of Universidad Nacional Autónoma de Honduras, UNAH.

For this educational intervention, developed during the first academic term of 2016, a teaching approach known as "spin-first" was adopted, through which the principles of modern quantum mechanics are introduced. 
Forefront computational tools for education were used, such as the open-source version of the platform Open edX, an application developed by Oregon State University (OSU) named SPINS to treat the subject interactively, several written and video tutorials based on student difficulties reported by worldwide known universities.

Among the main results obtained through a survey carried out, $80 \%$ of participants highlight great satisfaction, which considered the approach more enriching regarding their learning than others merely theoretical as the one offered by MIT through its MOOC platform edX. Further, a special evaluation revealed the formalism and fundamental concepts were well assimilated by the students. These results motivate to keep research ongoing for the teaching/learning area of this rigorous branch of physics, quantum mechanics.

\section{Keywords:}

Quantum mechanics, SternGerlach experiments, virtual learning environments, active engagement in STEM.

\section{Introducción}

La enseñanza de la mecánica cuántica en la actualidad a nivel global está cambiando y no es para menos, los conceptos que la disciplina requiere van más allá de nuestra intuición, la cual está construida con base en experiencias. Además, la matemática involucrada es amplia: teoría de grupos, álgebra lineal, ecuaciones diferenciales, análisis de Fourier, entre muchos otros tópicos especiales. Por esto y más es que la mecánica cuántica es una disciplina difícil de aprender (Styer, 1996) y es necesario fortalecer o cambiar el modelo tradicional de enseñanza-aprendizaje en la UNAH. Diversos estudios en el ámbito de la educación de la física apuntan hacia enfoques en los que el estudiante esté involucrado de manera activa en el aprendizaje, no solo escuchando de forma pasiva de quien sabe: el profesor. Se ha demostrado que con ello adquiere una mejor comprensión de los conceptos de la física (Falk, 2007). En ese sentido, herramientas computacionales se pueden utilizar de diversas maneras para que el estudiante se compenetre con la materia: cátedras apoyadas con visualizaciones en computadora, tutoriales interactivos, tutoriales en documento o video, laboratorios virtuales, entre muchas otras formas (David H. McIntyre, 2007).

En ese sentido, para el curso de Física Cuántica (MFS614) de la Maestría en Física en Ciudad Universitaria se implementó en modalidad b-learning un módulo que involucra al estudiante en su aprendizaje haciendo uso de herramientas computacionales de vanguardia y un enfoque pedagógico innovador que se utiliza en algunas universidades como Universidad Estatal de Oregon (OSU). En este, se usan experimentos de Stern-Gerlach para la enseñanza de la mecánica cuántica moderna. Zhu y Singh de la Universidad de Pittsburg, han reportado mejorías en la comprensión de los conceptos de la mecánica cuántica utilizando experimentos de Stern-Gerlach, basados en un simulador del experimento (SPINS) y contribuido -mediante tutoriales interactivos - a fortalecer la comprensión de este enfoque (Guangtian \& Chandralekha, 2011).

En este artículo se describe brevemente el enfoque pedagógico utilizado en el módulo 2 del curso de Física Cuántica -experimentos de Stern-Gerlach- y las herramientas computacionales escogidas para respaldar el curso hacia el aprendizaje activo del estudiante; no sin antes enmarcar el campo de estudio e importancia de la mecánica cuántica en la actualidad y su futuro.

Se describe y discuten los resultados obtenidos en el curso mediante una encuesta realizada en Google Forms y una evaluación especial diseñada para medir la comprensión de la temática.

\section{Importancia de innovar en la enseñanza de la mecánica cuántica}

La mecánica cuántica estudia el comportamiento de lo 
pequeño: moléculas, átomos, partículas subatómicas y sus interacciones. La Teoría de Newton del siglo XII no logró describir estos fenómenos; simplemente no predice correctamente los resultados experimentales. Lo mismo se puede decir de la teoría de Einstein -La Relatividad-, que abarca objetos cuya rapidez es comparable a la de la luz.

Si la teoría de Einstein o Newton funcionan para la descripción del mundo macroscópico que nos rodea, ¿por qué estudiar mecánica cuántica? Históricamente las motivaciones fueron tratar de explicar fenómenos que no podían entenderse con física clásica (física antes del siglo XX), tales como la radiación de cuerpo negro, el efecto fotoeléctrico y la dispersión de Compton. Sin embargo, en la actualidad, dada su probada capacidad de predicción, es mucho más que eso.

Sin la teoría cuántica, desarrollada a lo largo del último siglo, no habría electrónica como la conocemos hoy en día, los teléfonos celulares y tabletas no serían portátiles. Los láseres y sus diferentes aplicaciones -tal como la transferencia de datos mediante fibra óptica- no estarían disponibles. La comprensión del Universo estaría muy limitada, ya que las teorías que hoy en día son promesa para explicar todo se basan en la mecánica cuántica.
Asimismo, la ciencia natural que conocemos como Química ha tenido grandes avances dado el entendimiento a nivel subatómico que brinda la mecánica cuántica. El Modelo Estándar, quizá la compilación intelectual más importante del siglo pasado sienta sus bases en la mecánica cuántica.

La mecánica cuántica satisface la curiosidad innata por entender el universo que nos rodea y ha permitido desarrollar tecnología para la comodidad del ser humano. El futuro es prometedor: computadoras muchísimo más rápidas, imagenología para aplicaciones médicas, seguridad de la información, etc., son solo algunas aplicaciones en desarrollo que utilizan fundamentos de mecánica cuántica.

Las universidades del mundo, a través de sus diferentes carreras, tienen en sus currículos académicos asignaturas relacionadas con la enseñanza de este campo de la física. Para las ingenierías -particularmente aquellas que estudian lo relacionado con química, electricidad y magnetismo, electrónica y computadoras-, es conveniente el aprendizaje de la mecánica cuántica. Por supuesto, es una necesidad para quienes estudian el campo de la Física.

Actualmente en la UNAH, y de hecho en todo Honduras, el único departamento que sirve asignaturas de mecánica cuántica es la Escuela de Física de la UNAH en Ciudad Universitaria; sin embargo, no cabe duda de que en el futuro -tarde o temprano- las ingenierías a nivel nacional deberán incorporarla en sus planes. La enseñanza de la Física Cuántica en la UNAH, a nivel de pregrado consta de dos cursos que se sirven de manera tradicional. Todavía más, la enseñanza tradicional en la física contempla experimentación, pero muchas Escuelas de Física en América Latina, incluyendo Honduras, carecen de presupuesto necesario para la adquisición de equipos para experimentación de Física Cuántica. Por ello, es imperativo innovar: las simulaciones computacionales son una alternativa atractiva para suplir esta necesidad.

La forma en que se aprende, por supuesto varía de una persona a otra, pero existen en la actualidad medios y herramientas didácticas que, a todos, sin excepción, pueden ayudar a mejorar los aprendizajes y tener una mayor comprensión de un tema. Estudios muestran que la enseñanza mediante actividades computacionales puede ser tan o más efectiva que los métodos tradicionales (Steinberg, 2000; Zacharia, 2003).

En el siguiente apartado se presenta la metodología, el diseño general del módulo, y las herramientas pedagógicas 
Tabla 1: Estructura del Curso MFS614

\begin{tabular}{|c|c|c|}
\hline Módulo 1 (MIT edX) & Módulo 2 (Open edX DIE) & Módulo 3 \\
\hline $\begin{array}{l}\text { Mecánica Ondulatoria } \\
\text { (Schrödinger) }\end{array}$ & $\begin{array}{l}\text { Experimentos de Stern-Ger- } \\
\text { lach para sistemas de Espín }\end{array}$ & Momento Angular \\
\hline Espín 1/2 & $1 / 2$ y 1 & Átomo de Hidrógeno \\
\hline \multicolumn{3}{|l|}{ Álgebra Lineal } \\
\hline \multicolumn{3}{|l|}{$\begin{array}{l}\text { Brakets y Principio de Incer- } \\
\text { tidumbre }\end{array}$} \\
\hline $\begin{array}{l}\text { Duración: } 1 \text { mes y medio fe- } \\
\text { brero y marzo }\end{array}$ & $\begin{array}{l}\text { Duración: } 1 \text { mes } \\
\text { Abril }\end{array}$ & $\begin{array}{l}\text { Duración: } 2 \text { semanas } \\
\text { Mayo }\end{array}$ \\
\hline
\end{tabular}

Por Jonathan López con base en información proporcionada por Dr. Armando Euceda

y computacionales más importantes utilizadas para la enseñanza de la teoría de la mecánica cuántica en el curso de posgrado Física Cuántica.

Desarrollo de la intervención educativa innovadora

Para comenzar a describir el proceso innovador del módulo 2 de la asignatura de postgrado Física Cuántica, es importante referir que el contenido programático del curso de Física Cuántica (MFS614) de la Maestría de Física que ofrece la UNAH, consta de tres módulos, cuya temática se resume en la tabla 1.

La dinámica de desarrollo de la asignatura MFS614 durante el primer periodo académico 2016 fue la siguiente: el primer módulo, a cargo del profesor Armando Euceda, se desarrolló con el apoyo del MOOC impartido por el Massachusetts Institute of Technology a través en la plataforma edX. El módulo 2 en el cual se aplicó la innovación tema de este artículo, estuvo a cargo del profesor Jonnathan Andre López y se desarrolló en modalidad b-learning. Y el tercer módulo a cargo del profesor Armando Euceda.

El módulo 2 para MFS614 se diseñó y desarrolló en un aula virtual en la plataforma Open edX y el simulador SPINS para experimentos de Stern-Gerlach, aplicación desarrollada originalmente por Schroeder y Moore para Macintosh (D.V. Schroeder, 1993) y portado a Java por David McIntyre (David $H$. McIntyre, 2007).

En contraste a los cursos de mecánica cuántica tradicionales, la estructuración del Módulo 2 titulado "Experimentos de Stern-Gerlach”, consistió en varios elementos innovadores: desde el entorno físico hasta las herramientas computacionales, el principal instrumento fue el aula virtual en la plataforma Open edX instalada en el campus virtual de la UNAH por el equipo técnico de la Dirección de Innovación Educativa (DIE). Esta aula virtual contiene los demás elementos de apoyo pedagógico y temático para los estudiantes: recursos didácticos, visualizaciones computaciones, la aplicación SPINS, tareas, tutoriales en video, laboratorios virtuales y la evaluación final. La propuesta innovadora en modalidad b-learning, desde luego incluye momentos presenciales y trabajo educativo en el aula virtual. El módulo 2 se impartió durante el mes de abril de 2016, los viernes y sábados, con un total de 20 horas presenciales y 10 horas de trabajo individual en línea aproximadamente. Ambos ambientes demandan condiciones pedagógicas y tecnológicas que contribuyan a innovar el proceso de aprendizaje del módulo 2 de la asignatura Física Cuántica. 


\section{Ambiente físico}

Los ambientes físicos pueden ser muy importantes en el aprendizaje de los estudiantes e incluso en sus calificaciones finales. Con base en análisis estadístico, Suleman (2014) mostró que los estudiantes se sienten más motivados, interesados y atentos; además, muestran una mejor retentiva a la hora de aprender.

El espacio físico utilizado fue el aula de la Maestría de Física que tiene las siguientes características: espacio físico amplio con capacidad para unas 60 personas, con mobiliario educativo y equipo educacional y de cómputo que demandan los espacios educativos del siglo XXI: televisores (4), proyec34 tor de alta definición, mesas circulares (4), varias pizarras, computadoras con conexión a Internet disponibles para los estudiantes y acceso a Internet inalámbrico.

Sin este ambiente físico, sería difícil involucrar al estudiante en el aprendizaje activo, concepto conocido como active-engagement. Las mesas circulares fomentan la discusión en grupos y permiten a los estudiantes disponer de sus computadores portátiles. Este entorno es propicio ya que "promueve la interacción y sentido de comunidad que permite el aprendizaje formal e informal." (Cornell, 2002)

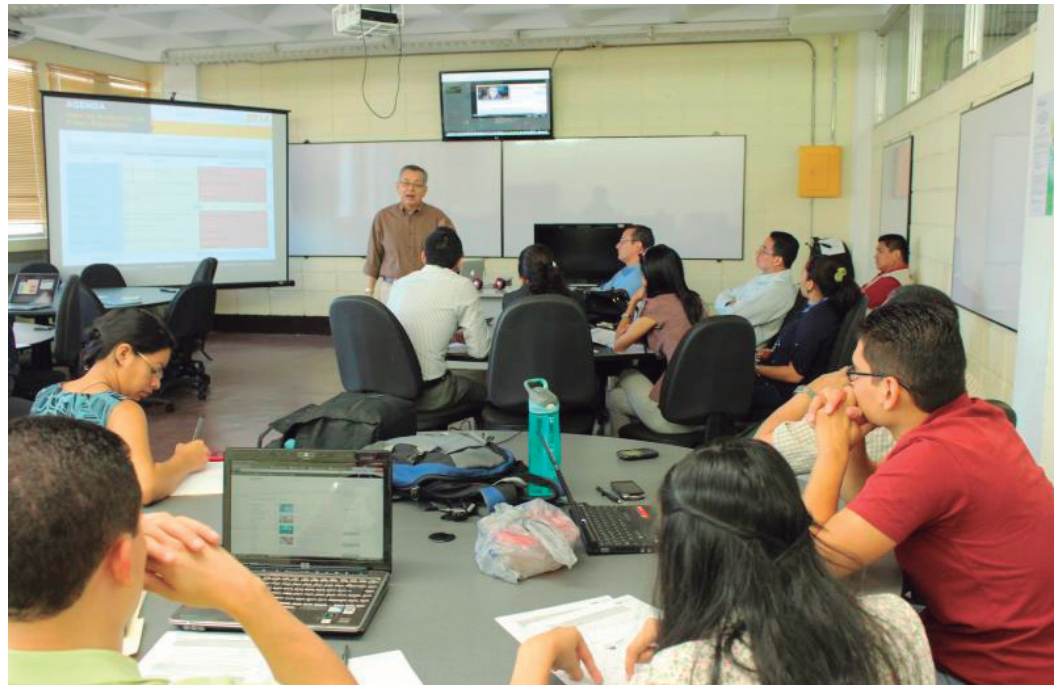

López, J. (2013) Aula de Maestría en Física.

Figura 1: Aula de la Maestría en Física utilizada para la asignatura de Física Cuántica.

\section{Herramientas computa- cionales utilizadas}

Aprovechando la amplia gama de herramientas computacionales e interactivas para la creación de entornos de aprendizaje virtuales, en la implementación del módulo 2 de la asignatura de Física Cuánticas se decidió el uso de las siguientes herramientas de cómputo: la plataforma Open edX y la aplicación SPINS.

La plataforma edX es una plataforma para la educación en línea, y una de las principales proveedoras de $\mathrm{MOOC}$ (Massive Open Online Course), surge en 2012 como iniciativa de la universidad de Harvard y el MIT.

Para instituciones educativas que desean montar cursos en línea utilizando esta plataforma, edX pone a disposición la versión Open edX. En la UNAH, esta plataforma de código abierto fue instalada como un campus abierto adicional al campus virtual institucional y se trabajó como proyecto piloto la asignatura de Física General FS-100 totalmente en línea, para estudiantes de las carreras de la Facultad de ingeniería.

La plataforma Open edX posee diversas herramientas para potenciar actividades de aprendizaje en las áreas de STEM, es decir los campos de las ciencias, matemáticas, ingenierías y tecnología. Permite codificación en XML, HTML5, Python y Perl, entre otras, haciéndola muy versátil para un instructor o docente con alto grado de formación en informática y programación, perfil que comúnmente poseen los profesores de Física, Matemáticas e Ingenierías.

Esta plataforma fue la elegida por las razones expuestas y fue el entorno con el cual el estudiante interactuó fuera y dentro del salón de clase. Es importante mencionar que el 


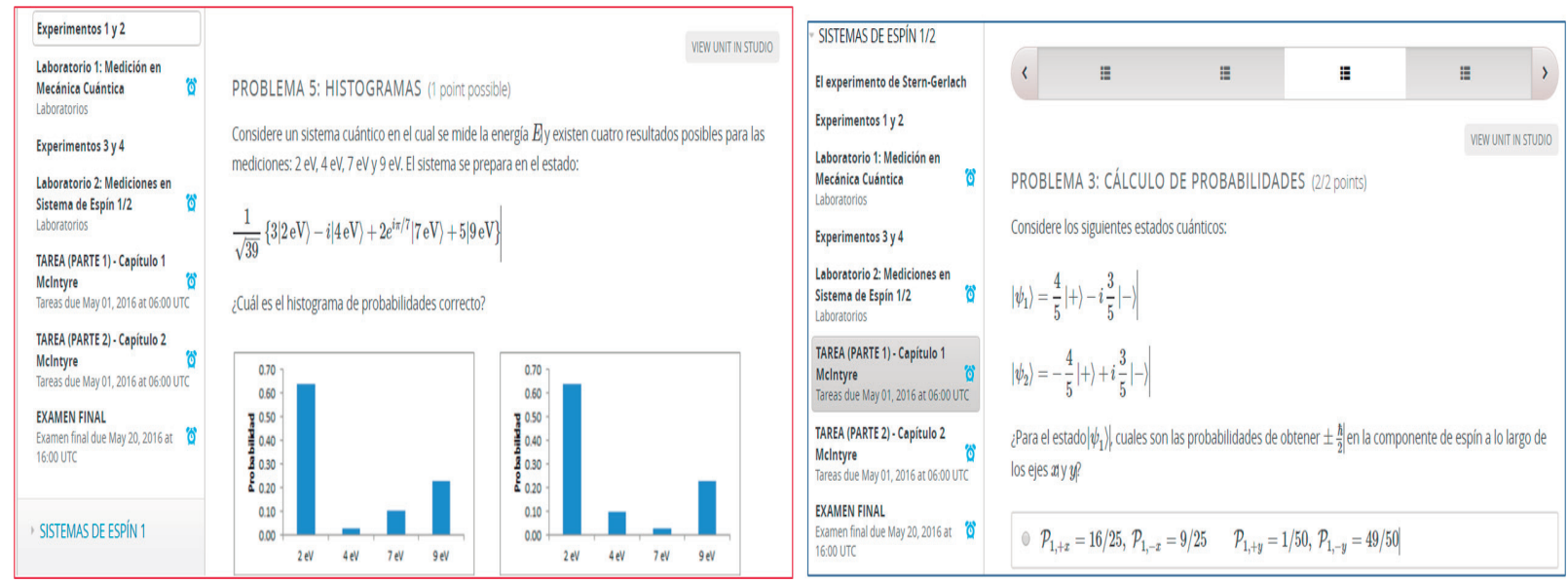

Figura 2. Capturas de Aula Virtual en Open edX

curso de Física Cuántica es el primer curso de posgrado en Honduras que está disponible en Open edX. Actualmente se está estructurando para que pueda ofrecerse completamente en línea (e-learning).

El aula virtual para el módulo 2 de Física Cuántica contiene además elementos tecnopedagógicos utilizados: desarrollo de la temática en HTML5 mediante documentos $\mathrm{y}$ videos, tutoriales escritos en Latex y en video utilizando el software ShowMe siguiendo el modelo de Khan Academy.

La comunicación con los alumnos en el aula virtual se realizó principalmente mediante video-mensaje elaborado con Camtasia Studio. El software utilizado para el desarrollo de la temática, esto es, el aprendizaje de los axiomas fundamentales de la mecánica cuántica mediante experimentos de Stern-Gerlach, fue el programa SPINS.

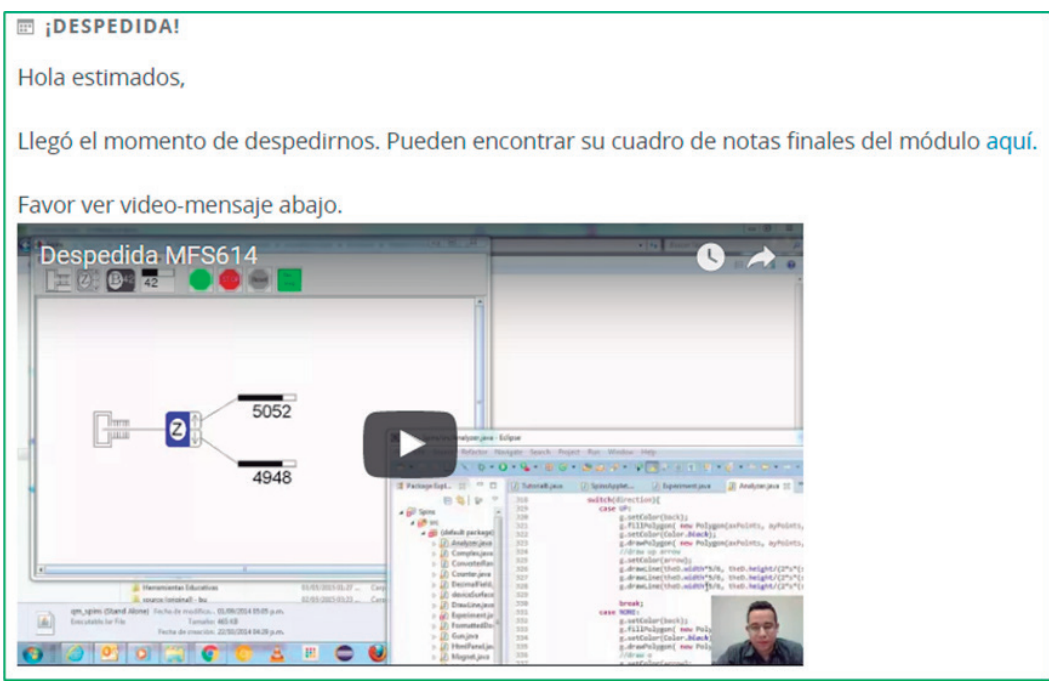

Figura 3: Captura de video-mensaje de despedida del curso. Se muestra en el fondo la aplicación SPINS en entorno de desarrollo Java.

\section{El uso de SPINS}

La Universidad Estatal de Oregon (OSU), en Estados Unidos, renovó en 2008 su diseño curricular para cursos avanzados de pregrado, y mecánica cuántica no fue la excepción. Entre las diferentes innovaciones realizadas en los cursos de Física Cuántica cabe destacar la incorporación de un software escrito en Java nombrado SPINS. La versión original fue escrita en Macintosh en 1993 por D.V. Schroeder. (David H. McIntyre, 2007).
Java es un lenguaje multiplataforma: sus aplicaciones corren en cualquier sistema operativo y funciona en la actualidad en $89 \%$ de las computadoras del mundo y más de 3 billones de dispositivos móviles (Oracle, 2016). En otras palabras, los estudiantes pueden sin problemas correr la aplicación en sus laptops o teléfonos móviles que casi siempre cargan consigo.

Esta aplicación permite realizar diferentes experimentos de Stern-Gerlach para el en-
35 2016 
tendimiento de los axiomas fundamentales de la mecánica cuántica. Además, concede al estudiante confirmar sus resultados teóricos mediante experimentación.

La aplicación SPINS consiste en una interfaz gráfica que simula los experimentos de Stern-Gerlach. La interfaz permite al usuario crear diversas configuraciones experimentales relacionadas con medición y dinámica cuántica. SPINS es una de las herramientas computacionales en mecánica cuántica más recomendadas y catalogada por expertos como "excelente" (E Dębowska, 2013).

Una captura de un experimento realizado durante el curso se muestra en la figura No. 3 .

\section{Diseño pedagógico}

E1 enfoque pedagógico utilizado para la enseñanza de la mecánica cuántica en el módulo 2 difiere notablemente de la enseñanza tradicional de la materia a nivel mundial. El famoso experimento llevado a cabo en 1,922 por Otto Stern y Walther Gerlach demostró que el momento angular intrínseco del electrón toma valores cuantizados. Experimentos de este tipo sumergen al estudiante a los nuevos aspectos de la mecánica cuántica, en relación con la física clásica.

Dos razones fundamentales resumen la justificación del uso de experimentos de
Stern-Gerlach (McIntyre, Quantum Mechanics, 2012):

I) Demuestra cómo la mecánica cuántica moderna funciona ilustrando sus postulados fundamentales.

II) Demuestra cómo se trabaja de manera práctica en la mecánica cuántica a través de notación de Dirac y formalismo matricial.

III) Algunas universidades han adoptado este enfoque de manera parcial, como el MIT, y otras presentan este enfoque como primer paso de estudio en mecánica cuántica; tal es el caso de la Universidad Estatal de Oregon (OSU). Este tratamiento se basa en presentaciones realizadas previamente por Feynman, Leighton y Sands; Cohen-Tannoudji, Diu, y Laloe; Sakurai; y Towsend (McIntyre, Quantum Mechanics, 2012).

E1 enfoque por sí solo supone un recurso invaluable en el aprendizaje, pero queda perfectamente complementado con el software SPINS discutido previamente. Con éste, los alumnos ponen sus «manos a la obra» e interactúan dentro y fuera del aula. Además, el instructor puede realizar demostraciones diversas en clase utilizando la aplicación.

Los momentos presenciales en aula de clase se orientaron a la exposición teórica de conceptos fundamentales mediante ayudas visuales y computacionales; discusión y aclaración de conceptos difíciles de com- prender reportados por Zhu y Singh (Guangtian \& Chandralekha, 2011) y, en menor grado dado el limitado tiempo, a actividades de aprendizaje activo que involucraban al estudiante. Estas últimas quedaban para el alumno en su tiempo fuera del aula de clase. Cada una de las cátedras y actividades, en salón o en aula virtual, tenía propósitos u objetivos definidos.

\section{Trabajo en el aula virtual}

En el aula Virtual se presentaron los temas del módulo en HTML5, documentos, videos, tareas en su mayoría ejercicios o preguntas conceptuales, que fueron cuidadosamente preparadas para cubrir las ideas esenciales y lograr que los estudiantes practicaran el formalismo. Open edX permite múltiples opciones para el diseño de las tareas y admite escritura directa en LaTeX, lo cual hace que las tareas sean visualmente atractivas para el estudiante.

En la mayor parte de los casos los alumnos pueden comprobar sus respuestas mediante la experimentación, vía SPINS, lo cual hace que el estudiante se sienta confiado y lo motiva a seguir practicando y explorando. Adicionalmente, dos laboratorios virtuales fueron propuestos con pequeños tutoriales previos en LaTeX y en video para que el alumno logrará una mejor comprensión de lo requerido. 


\section{Resultados obtenidos y percepción de los estu- \\ diantes}

Los resultados de esta innovación educativa en la asignatura de Física Cuántica se agrupan en tres componentes, el primero de diseño y de desarrollos educativos innovadores para el aprendizaje del módulo 2 en modalidad b-learning, como se describe en el apartado anterior. En segundo, el referido a la evaluación de los aprendizajes con la nueva metodología y herramientas tecnológicas de soporte. En tercer lugar, los referidos a la percepción de los estudiantes que cursaron la asignatura en mención y que se detallan a continuación.

Se refiere que el curso estaba constituido por 14 estudiantes, $50 \%$ de ellos profesores de grado de la UNAH para pregrado. Doce de ellos con jornada de trabajo de tiempo completo mientras que 2 lo hacían a medio tiempo. Según su percepción, el trabajo paralelo influye considerablemente en su aprendizaje dentro de la Maestría en Física. La mayor parte, el $57 \%$, por la naturaleza de su carrera de licenciatura, nunca había tomado cursos de física cuántica antes de entrar al curso de la maestría.

Estos estudiantes tomaron primero el curso ofrecido por el MIT edX 5.01.x, como Módulo 1 del curso. Durante cuatro semanas trataron la misma temática, pero de forma teórica, sin «experimenta-

\section{4. ¿Que opinión le merece la plataforma edX?}
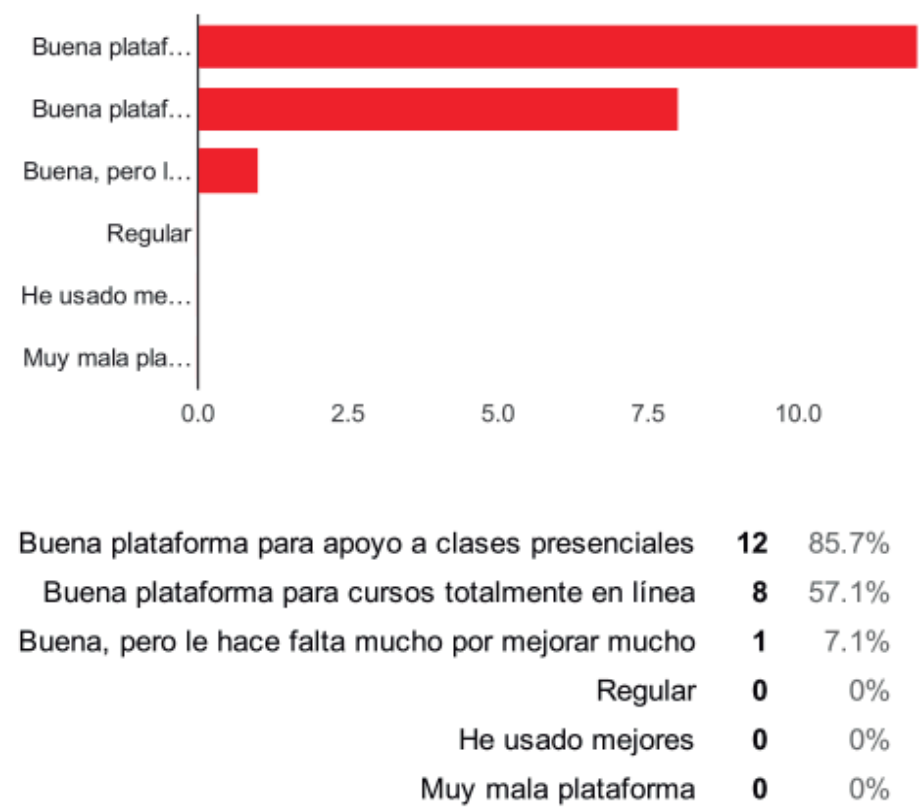

Figura 4. Valoración de la plataforma Open edX

20. Considera que el sotware SPINS tiene:

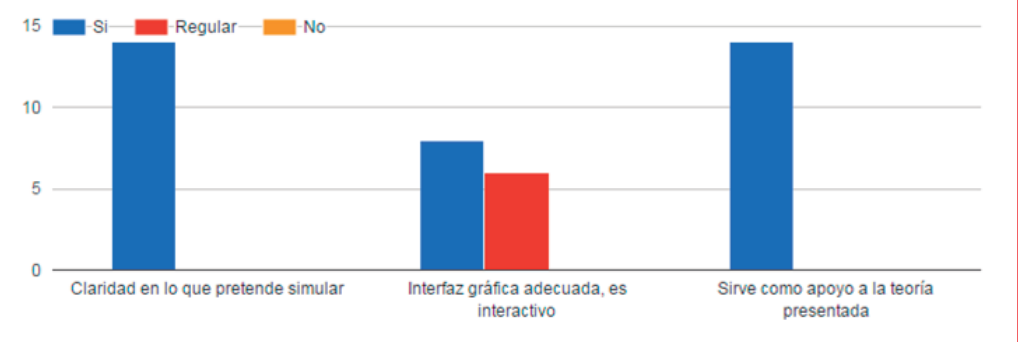

ción» virtual ni las actividades computacionales ofrecidas en el módulo 2. Ambos módulos fueron ofrecidos con Open edX como entorno virtual.

\section{Evaluación de los aprendi-} zajes

Se realizó una evaluación en el aula virtual Open edX para investigar la viabilidad de la metodología y herramientas utilizadas para lograr el aprendizaje de los alumnos, prepa- rada con base en conceptos usualmente difíciles de comprender.

La evaluación tomó como base dificultades percibidas a lo largo del Módulo y además las reportadas en la Universidad de Pittsburgh (Guangtian Zhu, 2011). El examen consistió en diez preguntas conceptuales rigurosas, cuidadosamente elaboradas, y tres problemas complejos. Cada 
ítem persigue objetivos específicos definidos a manera de cubrir todo el contenido propuesto. Los estudiantes podían hacer uso de SPINS para verificar sus soluciones, cuando aplicaba.

Los resultados fueron alentadores: el promedio obtenido fue del $96 \%$ en contraste con el $75 \%$ para el curso módulo 1 del MIT. Sus procedimientos (lápiz y papel) muestran que sus notas no fueron casualidad, sino que realmente aprendieron lo esperado a través de las innovaciones incorporadas en el módulo 2.

Percepción de las herramientas tecnológicas

38 Para conocer la percepción de los estudiantes sobre la plataforma Open edX y del software SPINS utilizados en el aula del módulo 2, se preparó una encuesta de 26 preguntas y los resultados son los siguientes:

En cuanto a las condiciones de estudio el $86 \%$ de los estudiantes consideró que la plataforma Open edX es una buena plataforma para modalidad b-learning como se ofreció el módulo 2 , mientras que $57 \%$ coincidieron que también lo es para la modalidad e-learning como cursaron el módulo 1 . El $7 \%$ de los participantes opinan que hay aspectos por mejorar (ver figura 4).

En cuanto al software SPINS, los estudiantes opinaron que esta aplicación tiene claridad en lo que pretende simular, pero para el $43 \%$ de ellos no es lo suficientemente interactivo y requiere mejorar su interfaz gráfica. Sin embargo, el 100\% coincidió en que logra su cometido: servir como apoyo a la teoría presentada (ver figura 5).

La totalidad de los estudiantes consideró que el Módulo 2 fortaleció sus conocimientos en Mecánica Cuántica, principalmente porque involucra experimentación. Según su percepción, el instructor también fue un factor importante para el éxito de su aprendizaje. Además, el 80\% consideró que este enfoque fue superior que el meramente teórico del MIT, mientras que un $20 \%$ opina que fue similar, salvo que en el MIT no hubo parte experimental. El $100 \%$ de los alumnos indicó que prefiere la modalidad semi-presencial (b-learning) con recursos y apoyo en línea mediante Open edX.

\section{Conclusiones y recomen- daciones}

La implementación del módulo 2 en el curso de Física Cuántica de Posgrado con elementos innovadores en cuanto al enfoque temático y pedagógico, así como el uso de herramientas computacionales como Open edX y el software SPINS, cuyo objetivo principal fue involucrar activamente al estudiante en el aprendizaje, se logró.

Los resultados obtenidos sugieren que el aprendizaje de la mecánica cuántica mejora con el enfoque innovador incorporado en el desarrollo del módulo 2 , innovaciones que deben ser consideradas para su incorporación a nivel de pregrado, ya sea como primer acercamiento a la mecánica cuántica (spin-first) previo al estudio de la mecánica ondulatoria, o bien como complemento experimental a los cursos actuales mediante la inclusión de laboratorios virtuales

Las preferencias de los estudiantes se inclinadas hacia aprendizaje b-learning con experimentación virtual en comparación con enfoques meramente teóricos en modalidad e-learning.

La evaluación final aplicada a los estudiantes mostró una sólida comprensión de los alumnos hacia los conceptos fundamentales y formalismo de los temas de la materia de la Física Cuántica abordados en el módulo 2.

Esta experiencia de innovación educativa estimula a que se sigan realizando intervenciones pedagógicas innovadoras con sus respectivas valoraciones del impacto en los aprendizajes, tanto a nivel de pregrado como posgrado, para las diferentes asignaturas que sirve la Escuela de Física.

\section{Referencias}

Cornell, P. (2002). The impact of changes in teaching and 
learning on furniture and the learning environment. New Directions for Teaching and Learning, 33-42.

D.V. Schroeder, T. M. (1993). A computer-simulated Stern-Gerlach laboratory. Am. J. Phys., 798-805.

David H. McIntyre, J. T. (2007). Integrating computational activities into the upper-level Paradigms in Physics curriculum at Oregon State University. American Journal of Physics.

E D囚bowska, R. G. (2013). Report and Recommendations on Multimedia Materials for Teaching and Learning Quantum Physics . European Journal of Physics, Volume 34, Number 3, 5 .

edX. (2012).edX Organization. Recuperado el 04 de 09 de 2016, de https://www.edx. org/about-us

Falk, J. (2007). Students' depictions of quantum mechanics:a contemporary review and some implications.

Guangtian, Z., \& Chandralekha, S. (2011). Improving students' understanding of quantum mechanics via the Stern-Gerlach experiment. Am. J. Phys.

López, J. (2016). Encuesta MFS614. Tegucigalpa,
Honduras. Recuperado de https://goo.g1/MeBWTE

McIntyre, D. H. (2012). Quantum Mechanics. En D. H. McIntyre, Quantum Mechanics (pág. 1). Oregon: Pearson.

McIntyre, D. H. (2012). Quantum Mechanics. En D. H. McIntyre, Quantum Mechanics (pág. XV). San Francisco: Pearson.

Oracle. (2016). Acerca de: Java. Recuperado de https:// www.java.com/es/about/

Steinberg, R. (2000). Computers in teaching science: to simulate or not to simulate. Am. J. Physics, S37-S41.

Styer, D. (1996). Common misconceptions regarding quantum mechanics. Am. J. Phys., 31-34.

Suleman, Q. (2014). Effects of Classroom Physical Environment on the Academic Achivement. International Journal of Learning $\&$ Development.

Z. Zacharia, O. A. (2003). The effects of the interactive computer-based simultation prior to performing laboratory inquiry-based experiment on students' conceptual understanding on physics. Am. J. Physics, 618-629. 